\title{
Ultracold Nonreactive Molecules in an Optical Lattice: Connecting Chemistry to Many-Body Physics
}

\author{
Andris Doçaj, ${ }^{1,2}$ Michael L. Wall, ${ }^{3}$ Rick Mukherjee, ${ }^{1,2}$ and Kaden R. A. Hazzard ${ }^{1,2, *}$ \\ ${ }^{1}$ Department of Physics and Astronomy, Rice University, Houston, Texas 77005, USA \\ ${ }^{2}$ Rice Center for Quantum Materials, Rice University, Houston, Texas 77005, USA \\ ${ }^{3}$ JILA, NIST and University of Colorado, Boulder, Colorado 80309-0440, USA \\ (Received 22 December 2015; revised manuscript received 24 February 2016; published 30 March 2016)

\begin{abstract}
We derive effective lattice models for ultracold bosonic or fermionic nonreactive molecules (NRMs) in an optical lattice, analogous to the Hubbard model that describes ultracold atoms in a lattice. In stark contrast to the Hubbard model, which is commonly assumed to accurately describe NRMs, we find that the single on-site interaction parameter $U$ is replaced by a multichannel interaction, whose properties we elucidate. Because this arises from complex short-range collisional physics, it requires no dipolar interactions and thus occurs even in the absence of an electric field or for homonuclear molecules. We find a crossover between coherent few-channel models and fully incoherent single-channel models as the lattice depth is increased. We show that the effective model parameters can be determined in lattice modulation experiments, which, consequently, measure molecular collision dynamics with a vastly sharper energy resolution than experiments in a free-space ultracold gas.
\end{abstract}

DOI: 10.1103/PhysRevLett.116.135301

Introduction.-The recent production of ultracold ground state molecules opens up far-ranging possibilities for quantum many-body physics. These possibilities stem from properties unavailable to atoms, including strong, long-range electric dipole-dipole interactions and a rich rotational and vibrational structure. Relying on these properties, ultracold molecules can be used for quantum simulation of strongly interacting systems [1-3], quantum information processing [4], quantum metrology, and exploring chemistry in the quantum regime [5-11]. The first achieved [12] and most explored [13-22] ultracold molecule, $\mathrm{KRb}$, reacts rapidly, $\mathrm{KRb}+\mathrm{KRb} \rightarrow \mathrm{K}_{2}+\mathrm{Rb}_{2}$ [18], as do half of the alkali metal dimers [23]. Experiments are underway to cool many of these reactive species [24-27]. Even though reactions offer exciting insights into quantum chemical kinetics and stereodynamics [17], they limit the cloud lifetime. Although reactions are sometimes irrelevant, as for quantum spin models [3,21,28-31], and sometimes can be suppressed [32-35], they can prevent accessing situations where translational motion of the molecules is important.

Over the last year experiments have produced ultracold nonreactive molecules (NRMs) [36-40], and many other experiments are progressing with molecules that are nonreactive or whose reactivity is unknown [41-52]. Such molecules are expected to have long lifetimes, but to take advantage of these lifetimes we must understand the molecular interaction properties. It has recently been argued that the collisions of NRMs are much more complex than for atoms due to an extraordinarily high density of internal states at short range and low collision energies [53-55]. This complexity persists in the absence of an electric field and for homonuclear molecules [56-62]. Although many interesting scenarios for future NRM experiments involve optical lattices, the complexity of molecular collisions implies that the single-channel pseudopotential approach leading to the Hubbard model description of atoms in optical lattices $[63,64]$ will rarely apply to NRMs. Hence, an alternative approach is required to derive effective lattice models describing NRMs.

In this Letter, we provide a framework for deriving effective many-body models for NRMs in deep optical lattices or other tight traps, such as optical tweezers $[65,66]$. This framework combines transition state theory [67], random matrix theory (RMT) [68,69], and quantum defect theory [70-72] and accounts for the separation of short- and long-range scales shown in Fig. 1. We first solve the problem of two NRMs in a single site of an optical lattice that are coupled to a dense collection of short-range collision complexes. This on-site solution is coupled to other lattice sites (via tunneling) to obtain a full lattice model. Our method resembles those used to derive models of two-channel Feshbach resonances [74-77], but the physics differs significantly. Strikingly, despite the complexity of this system, we show that the model parameters depend universally on only two molecular properties: the density of bound states at zero collision energy $\rho_{b}$ and the van der Waals length $R_{\mathrm{vdW}}=\left(2 \mu C_{6}\right)^{1 / 4}$ for the potential $-C_{6} / R^{6}$, where $\mu$ is the two-molecule reduced mass $(\hbar=1$ throughout). Furthermore, we show that the model parameters can be experimentally characterized with lattice modulation spectroscopy. While our quantitative analysis uses a specific collisional model, our general procedure is broadly applicable. 


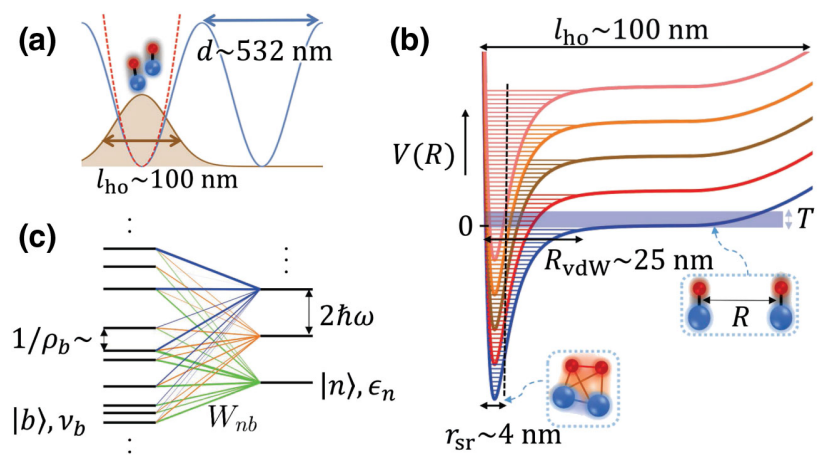

FIG. 1. Confined nonreactive molecules. (a) NRMs in a deep optical lattice (solid) idealized by a harmonic trap (dashed). (b) At large intermolecular separations $R$, NRMs experience only the harmonic trap, but for $R \lesssim r_{\mathrm{sr}}$ they couple to the numerous collisional complexes associated with excited rotational and vibrational interaction channels. Numbers are for RbCs [73]. (c) Harmonic oscillator states $|n\rangle$ with energy $\epsilon_{n}$, and bound state complexes $|b\rangle$ with energy $\nu_{b}$ and density of states $\rho_{b}$ are coupled by $W_{n b}$.

Formalism.-Figure 1 shows a schematic of NRMs in a deep optical lattice and our theoretical approach. A single site containing two NRMs is approximated by a harmonic well of angular frequency $\omega$ [Fig. 1(a)], which is quantitatively accurate for on-site properties in a deep lattice [78]. The trap quantizes motion to harmonic oscillator states that spread out over the harmonic oscillator length $l_{\mathrm{ho}}=\sqrt{1 /(\mu \omega)} \sim 100 \mathrm{~nm}$. There are a vast number of internal degrees of freedom, e.g., vibrations and rotations, each associated with its own interaction potential (channel). Each channel may support several bound states, i.e., bimolecular collisional complexes, at short range, $r_{\mathrm{sr}} \sim$ $4 \mathrm{~nm}$ [Fig. 1(b)]. Although at intermolecular separations $R \gg r_{\text {sr }}$ the closed channels-defined by having $R \rightarrow \infty$ energies vastly larger than the collision energy-are negligible, the bound states couple to the open channel at $R \lesssim r_{\text {sr }}$. Despite this separation of length scales, openchannel-dominated spatially extended states, e.g., halo states near atomic Feshbach resonances, are captured by our model. Two NRMs confined to a site of an isotropic 3D optical lattice are described by $H=H_{\mathrm{c} . \mathrm{m} \text {. }}+H_{\text {rel }}$, where the center-of-mass (c.m.) Hamiltonian is $H_{\text {c.m. }}=\omega\left(2 n_{\text {c.m. }}+\right.$ $\left.\ell_{\text {c.m. }}+3 / 2\right)$ with $n_{\text {c.m. }}$ and $\ell_{\text {c.m. }}$ the c.m. principal and angular momentum quantum numbers, and the relative coordinate Hamiltonian for the $\ell=0 s$-wave states is

$H_{\mathrm{rel}}=\sum_{n} \epsilon_{n}|n\rangle\left\langle n\left|+\sum_{b} \nu_{b}\right| b\right\rangle\langle b|+\sum_{b n}\left(W_{n b}|n\rangle\langle b|+\right.$ H.c. $)$,

with $\epsilon_{n}=(2 n+3 / 2) \omega$ the energy of harmonic oscillator state $|n\rangle, \nu_{b}$ the energy of bound state $|b\rangle$, and $W_{n b}$ the coupling of harmonic oscillator state $|n\rangle$ to bound state $|b\rangle$ [79]. Figure 1(c) displays the structure of this model.
Although higher partial waves contribute in principle, these are suppressed at low energy by Wigner threshold laws [54]. Because of the separation of scales $\left\{r_{\mathrm{sr}}, R_{\mathrm{vdW}}\right\} \ll l_{\text {ho }}$, we can approximate the bound states as delta functions, in which case the couplings are $W_{n b}=w_{b} M_{n} / l_{\text {ho }}^{3 / 2}$ with $M_{n}=\sqrt{\Gamma(n+3 / 2) / \Gamma(n+1)}, \Gamma(x)$ the gamma function; the constants $w_{b}$, which will be determined below, are independent of $n$ and the trap parameters. The Supplemental Material describes how to regularize divergences in this naive zero-range coupling approximation to obtain the correct physical limit [80].

Although Eq. (1) exactly describes two NRMs in a harmonic trap when $\left\{r_{\mathrm{sr}}, R_{\mathrm{vdW}}\right\} \ll l_{\mathrm{ho}}$, determining the $\nu_{b}$ and $w_{b}$ from an ab initio microscopic model of interacting NRMs is extraordinarily difficult. Similarly, measuring the parameters is infeasible: in contrast to simple atoms such as the alkalis, in which the density of short-range resonant states $\rho_{b}$ is small enough that individual resonances are easily resolved, NRMs are predicted to have $\rho_{b}$ so large that individual resonances are unresolvable at any reasonable temperature [53-55]. Hence, the full characterization of the interactions is expected to be beyond the reach of both current theory and experiment.

Instead of an $a b$ initio model of the interactions at short range, we use a simple-yet realistic and potentially accurate-parametrization to obtain the $\nu_{b}$ and $w_{b}$. This interaction model is essentially a Hamiltonian reformulation of the approach introduced in Refs. [53,54]. First, we apply random matrix theory (RMT), which is expected to be valid in the often-relevant case where molecular collisions are chaotic [55,84-89]. Specifically, the $\nu_{b}$ are the eigenvalues of matrices sampled from the Gaussian orthogonal ensemble (GOE). The GOE probability distribution is $P_{H}\left(H_{b}\right)=B e^{-\operatorname{Tr} H_{b}^{2} / 2 \sigma^{2}}$ over $H_{b}$ in the set of $N_{b} \times N_{b}$ real symmetric matrices, $B$ is a normalization factor, and $\sigma=\sqrt{N_{b}} /\left(\pi \rho_{b}\right)$ is chosen to match the molecule's $\rho_{b}$ for $N_{b} \rightarrow \infty$. The coupling $w_{b}$ 's probability distribution is $P_{w}\left(w_{b}\right)=C e^{-w_{b}^{2} / 2 \sigma_{w}^{2}}$, where $C$ is a normalization constant. In particular, we determine $\sigma_{w}$ for a given molecule by matching Eq. (S3)'s free space $(\omega \rightarrow 0)$ bound state decay rate to the physical decay rate. The former is obtained by Fermi's golden rule. The latter is approximated by combining Rice-Ramsperger-KasselMarcus (RRKM) transition-state theory to account for the decay rate at $R \sim r_{\mathrm{sr}}$ with quantum defect theory (QDT) to describe propagation in the van der Waals tail of the intermolecular potential $[53,54,90]$. RRKM is a standard chemical approximation whose core assumption is that the molecules' configurations are in equilibrium until they cross a reaction surface, which then is never recrossed [67]. QDT is an exact treatment of the potential tail that is crucial to obtain the Wigner threshold laws. This procedure, explained in more detail in the Supplemental Material [80], yields $\sigma_{w}=2 \sqrt{\left(R_{\mathrm{vdW}} / \pi^{3} \mu \rho_{b}\right)} \Gamma(3 / 4)$; remarkably, $\sigma_{w}$ depends only on $R_{\mathrm{vdW}}$ and $\rho_{b}$. 
Spectrum of two NRMs in a lattice site.-We numerically solve Eq. (1) using the $\nu_{b}$ and $W_{n b}$ described above to obtain the eigenvalues $E_{\alpha}$ and eigenstates $|\alpha\rangle$ [80]. Figure 2 displays the behavior of $E_{\alpha}$ and $|\alpha\rangle$ using parameters similar to current estimates for $\mathrm{RbCs}$ [53,73], $\rho_{b} \approx 0.5 / \mathrm{nK} \approx(2 \pi \times 20 \mathrm{~Hz})^{-1}$, and $R_{\mathrm{vdW}} \approx 25 \mathrm{~nm}$, though we stress that our model applies to any NRM. Figure 2(a) shows the spectrum for $H_{\text {rel }}$ as a function of harmonic oscillator frequency $\omega$, neglecting the c.m. energy common to all states. Harmonic oscillator energies increase with $\omega$, while short-range bound state energies are independent of $\omega$. For a given $\omega$, most of these eigenstates can be ignored since only those with appreciable open channel weight will be accessed experimentally. Therefore, the results are more informative if one sets the opacity of a point associated with $|\alpha\rangle$ to its weight on the open channel (trap states) $\mathcal{O}_{\alpha} \equiv \sum_{N, M}\left|O_{\alpha ; N, M}\right|^{2}$, where $O_{\alpha ; N, M} \equiv$ $\langle\alpha \mid N, M\rangle$ is the overlap of eigenstate $\alpha$ with the twoparticle open-channel state $|N, M\rangle$ labeled by the first and second particle's harmonic oscillator states, $N$ and $M$. Figure 2(b) displays the eigenstates for $\omega \lesssim 2 \pi \times 500 \mathrm{~Hz}$ weighted in this fashion, showing that most bound states
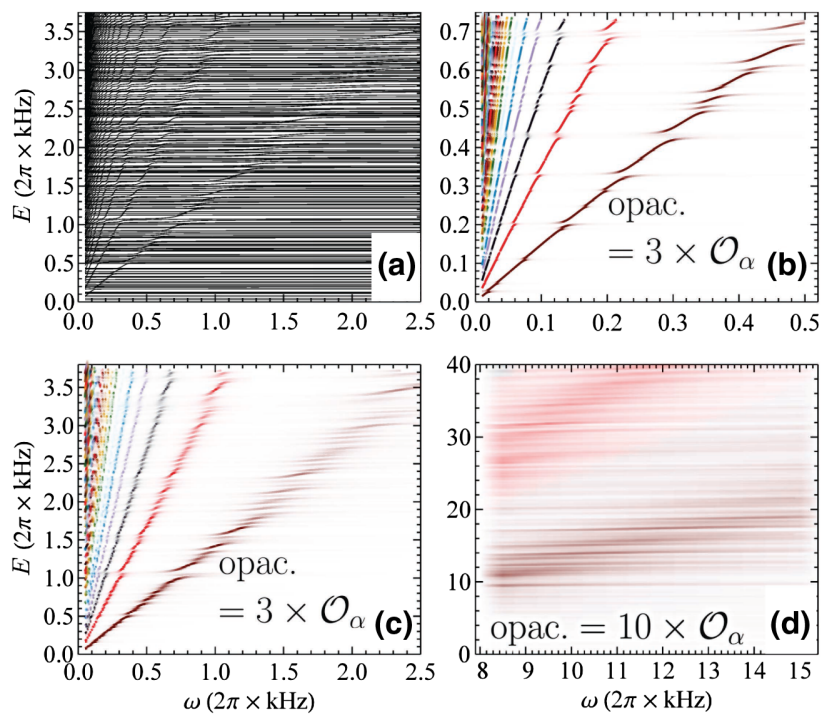

FIG. 2. States of two NRMs in a harmonic trap. (a) Eigenvalues vs trap frequency $\omega$ for RbCs. Bound states $|b\rangle$ (roughly horizontal) hybridize with harmonic oscillator states $|n\rangle$ (roughly diagonal). (b)-(d) By setting the opacity of a point to the associated eigenstate's open-channel probability $\mathcal{O}_{\alpha}$, the states relevant to the lattice model emerge from panel (a)'s tangle of lines. The notation opacity $=X \times O_{\alpha}$ means that we set the opacity of a point representing the eigenstate $\alpha$ (at a certain $\omega$ ) to $X$ times $O_{\alpha}$. (b) Weak trap, isolated resonances. (c) Intermediate lattice depths, rich structure of resonances coupled to harmonic oscillator states. (d) Deep trap, universal dissipative limit: resonances merge into a broad near-continuum. All of these regimes are experimentally relevant even though for RbCs in an isotropic 3D lattice the isolated resonance limit occurs at $\omega$ for which the harmonic oscillator approximation breaks down. are uncoupled from the trap states in this (small- $\omega$ ) isolated resonance frequency regime. Here NRMs are described by single- or few-channel models, just like atoms. In contrast to free space where the spread in energy is set by the temperature $k_{B} T$, and where, since $k_{B} T \gg \rho_{b}^{-1}$, many collisional complexes are coupled, the trap states' energies are precisely quantized and couple significantly only to a single collisional complex. Figure 2(d) displays the spectrum in deep traps $\omega \sim 2 \pi \times 15 \mathrm{kHz}$, which corresponds to trap depths similar to those used in common optical lattice experiments. The $W_{n b} \propto l_{h o}^{-3 / 2}$ are larger for the larger $\omega$ and so couple a broader energy range of collisional complexes. Because $W_{n b} \ll \omega$, there is still little mixing between the open channel states themselves for this $\omega$. However, many overlapping resonances couple to each $|n\rangle$, resulting in a smeared near-continuum of levels which we call the (large- $\omega$ ) universal dissipative limit, for reasons clarified later. Figure 2(c) shows the open-channel weighted spectrum for intermediate trap depths, $\omega \sim 2 \pi \times 2 \mathrm{kHz}$. In many ways this is the most novel regime, with a rich structure of nonisolated, but not completely overlapping, resonances.

We expect that all of these regimes are experimentally accessible. For RbCs, the universal dissipative limit and some of the intermediate regime occur where the harmonic oscillator approximation (and single-band, tight binding approximation for the lattice model presented below) will typically be valid, $\omega \gtrsim 5 \mathrm{kHz}$. In contrast, we present the isolated resonance limit mainly for its relevance to other NRMs. For NRMs with a smaller $\rho_{b}$, as expected for lighter NRMs (larger rotational constants) such as $\mathrm{NaK}[38,39]$, or smaller $R_{\mathrm{vdW}}$, as predicted for a range of molecules in Ref. [91], the crossover will occur at larger, more accessible, $\omega$. In particular, the crossover occurs when $W_{n b} \rho_{b} \sim 1$, equivalent to, using our earlier expressions, $\left(\omega \rho_{b}\right)^{3 / 4} \sqrt{R_{\mathrm{vdW}}\left(\mu / \rho_{b}\right)^{1 / 2}} \sim 1$.

Full lattice model. - Knowing the two-particle single-site solution, we couple sites to determine the effective lattice model $H_{\text {latt }}$ valid when at most two molecules per site are relevant. This regime describes a broad range of manybody experiments. Describing triply occupied (or higher) sites requires solving the numerically challenging threebody (or more) analog of Eq. (1) and will lead to new terms in the effective Hamiltonian, such as three-body interactions. The effective model for bosons is

$H_{\text {latt }}=-t \sum_{\langle i, j\rangle} c_{i}^{\dagger} c_{j}+\sum_{i}\left(\sum_{\alpha} U_{\alpha} n_{i, \alpha}+(3 / 2) \omega n_{i}\right)$.

Adapting this to fermions is straightforward under the additional assumption that internal (e.g., hyperfine and rotational) energy differences are much larger than collision and interaction energies. Equation (2) harbors two new features compared to the usual Bose-Hubbard model. (i) There are multiple interaction channels $\alpha$ with 
interaction energy $U_{\alpha}=E_{\alpha}-3 \omega / 2$, where $E_{\alpha}$ is the eigenenergy of $H_{\text {rel }}$. (ii) $c_{i}^{\dagger}$ and $c_{i}$ are modified from the usual creation or annihilation operators: $c_{i}^{\dagger}|\mathrm{vac}\rangle=|0\rangle_{i}$, $c_{i}^{\dagger}|0\rangle_{i}=\sum_{\alpha} \sqrt{2} O_{\alpha ; 0,0}|\alpha\rangle_{i}$, and $c_{i}^{\dagger}|\alpha\rangle_{i}=0$, where $|0\rangle_{i}$ is the site- $i$ single-particle ground state, i.e., $|N=0\rangle_{i}$. We have defined $n_{i, \alpha}=|\alpha\rangle_{i}\left\langle\left.\alpha\right|_{i} \text { and } n_{i}=\mid 0\right\rangle_{i}\left\langle\left. 0\right|_{i}+2 \sum_{\alpha} n_{i, \alpha}\right.$. Equation (2) is a many-channel generalization of Refs. [74,92]. We have ignored the collisional complexes' tunneling $t_{\mathrm{cc}} \ll t$ because they have approximately twice the molecules' polarizability and mass, and also ignored other terms that scale as $t_{\mathrm{cc}}$, such as molecules and complexes exchanging sites [92]. Equation (2) makes no assumption that the $|\alpha\rangle$ occupy only the lowest band; we require only that singly occupied sites'molecules reside in the lowest band. Figure 3(a) shows the $U_{\alpha}$ as a function of $\omega$. For each $\omega$, we plot the $U_{\alpha}$ for $\alpha$ that have $O_{\alpha}>0.2$. For the shown range of $\omega$, one or two channels typically are relevant.

Lattice modulation spectroscopy.-The interaction and overlap parameters $U_{\alpha}$ and $O_{\alpha ; 0,0}$ appearing in the lattice model Eq. (2) can be characterized, both theoretically and experimentally, via lattice modulation spectroscopy [93-95] as illustrated in Fig. 3(b). In this procedure, the
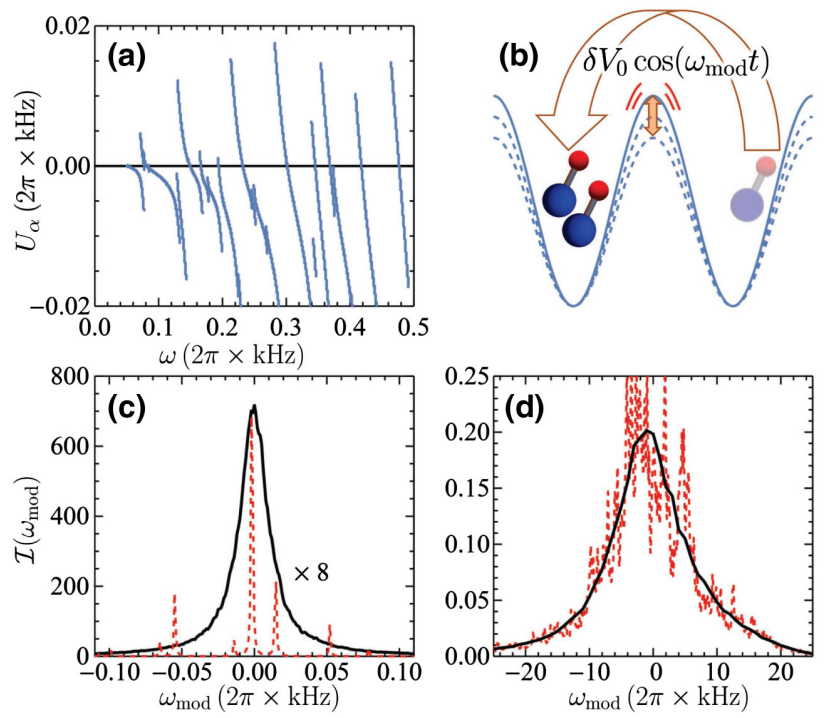

FIG. 3. Effective lattice model parameters and experimental characterization. (a) Interaction energies $U_{\alpha}$ versus $\omega$ for $\alpha$ 's that have weight on the trap ground state $\left|O_{\alpha ; 0,0}\right|^{2}>0.2$. (b) Lattice modulation spectrum $I\left(\omega_{\bmod }\right)$ : one slightly modifies the lattice depth at frequency $\omega_{\text {mod }}$ and measures the induced tunneling, for example the rate of change of double occupancies. This probes the lattice model parameters. (c) $I\left(\omega_{\bmod }\right)$ for $\omega=2 \pi \times 250 \mathrm{~Hz}$ for one realization of the random parameters (dashed line), and averaged over 10000 realizations (solid line). (d) Same as (c), but for $\omega=2 \pi \times 15 \mathrm{kHz}$, in the universal dissipative limit, and with the solid line averaging 50 realizations. Unlike the isolated resonance limit, $I\left(\omega_{\text {mod }}\right)$ "self-averages": one realization approaches the averaged spectrum and is thus independent of molecular details. depth of the lattice potential is modulated periodically with frequency $\omega_{\text {mod }}$, which induces tunneling from a noninteracting configuration where molecules are on neighboring lattice sites into an interacting configuration with two molecules on the same site. For weak modulation the response is given by linear response theory and we find a spectrum proportional to

$$
I\left(\omega_{\mathrm{mod}}\right)=\sum_{\alpha} \frac{\left|O_{\alpha ; 0,0}\right|^{2}}{\omega_{\mathrm{mod}}-U_{\alpha}+i 0^{+}},
$$

where $0^{+}$is a positive infinitesimal. Figures 3(c) and 3(d) show $I\left(\omega_{\text {mod }}\right)$ for different trap frequencies $\omega$. The dashed red curves are for a single realization of the RMT parameters, while the solid black curves average over many realizations. In the isolated resonance regime [Fig. 3(c)], well-separated peaks appear for each eigenstate $|\alpha\rangle$ with amplitude determined by $|\alpha\rangle$ 's weight on the ground harmonic oscillator state, $\left|O_{\alpha ; 0,0}\right|^{2}$, and the physics is well described by a few-channel model. For sufficiently small $\omega$, a single peak would dominate, and a one-channel model describes the physics. In this limit, the exact locations of the resonances are random, but can be predicted statistically. In the universal dissipative limit [Fig. 3(d), large $\omega$ ], many resonances smear together to give a continuous curve. For sufficiently large $\omega$ (but still small enough that band mixing is negligible), the spectrum for a single realization approaches the average over many realizations. Moreover, this spectrum approaches a Lorentzian with width $\gamma$; in fact, Eq. (2) reduces to a single channel model again, but with imaginary $U=-i \gamma$. This is consistent with studies of elastic collisions of NRMs in free-space [54], where the sticking rate due to the high density of states of the molecular complexes appears as a loss rate obtained when including a full absorbing, complex potential at short range. Although the interactions become fully incoherent, and thus in some sense classical, the tunneling remains fully coherent and quantum. In between these limits is the most novel regime, where several channels contribute but not so many that they can be described by an incoherent single-channel model. In closing, we stress that Eq. (2) holds more generally than our specific collision model, meaning that a model of the same form describes the system even when the RMT and RRKM approximations are invalid as long as the separation of scales in Fig. 1 is justified, and the spectra $I\left(\omega_{\bmod }\right)$ give a direct way of characterizing the lattice model parameters.

Conclusion.-We have put forth a framework for deriving effective lattice models for NRMs in optical potentials. We elucidated the models' structure and how to probe their parameters experimentally. We found a crossover between a coherent one-channel model (conventionally used to describe atoms) for weak traps to an incoherent Markovian single-channel model (often used to describe reactive molecules) for deep traps. In between, novel 
multichannel models emerge, whose many-body physics is an exciting frontier to explore.

Our results also show that optical lattice experiments can probe chemical properties: the bimolecular complexes. Unlike thermal gas measurements, which probe a range of energies set by the temperature, molecules in a trap have a tunable, exactly quantized energy. Thus NRMs in a lattice or optical tweezer array $[96,97]$ provide a high-energyresolution "chemical complex microscope" to probe the complexes' energies and couplings.

Clear next steps are to explore more elaborate collisional models and ways to control the interactions, e.g., via electric fields [98]. Finally, we note that our methods apply to other systems, such as lanthanide atoms [85,86], whose $\rho_{b}$ lies between molecules and alkali atoms.

We thank John Bohn, Brandon Ruzic, Svetlana Kotochigova, Michael Foss-Feig, Ana Maria Rey, Daniel Sheehy, Shah Alam, and Ian White for useful discussions. K. R. A. H. acknowledges the Aspen Center for Physics for its hospitality while part of this work was performed. This work was supported with funds from the Welch foundation, Grant No. C-1872. M. L. W. acknowledges support from the NRC postdoctoral fellowship program.

A. D. and M. L. W. contributed equally to this work.

*kaden.hazzard@gmail.com

[1] L. D. Carr, D. DeMille, R. V. Krems, and J. Ye, New J. Phys. 11, 055049 (2009).

[2] M. A. Baranov, M. Dalmonte, G. Pupillo, and P. Zoller, Chem. Rev. 112, 5012 (2012).

[3] M. L. Wall, K. R. A. Hazzard, and A. M. Rey, From Atomic to Mesoscale: The Role of Quantum Coherence in Systems of Various Complexities (World Scientific, Singapore, 2015), Chap. 1.

[4] D. DeMille, Phys. Rev. Lett. 88, 067901 (2002).

[5] G. Quéméner and P. S. Julienne, Chem. Rev. 112, 4949 (2012).

[6] P. F. Weck and N. Balakrishnan, J. Phys. B 39, S1215 (2006).

[7] N. Balakrishnan and A. Dalgarno, Chem. Phys. Lett. 341, 652 (2001).

[8] M. Tomza, Phys. Rev. Lett. 115, 063201 (2015).

[9] O. Dulieu and C. Gabbanini, Rep. Prog. Phys. 72, 086401 (2009).

[10] M. L. González-Martínez, O. Dulieu, P. Larrégaray, and L. Bonnet, Phys. Rev. A 90, 052716 (2014).

[11] R. V. Krems, Phys. Chem. Chem. Phys. 10, 4079 (2008).

[12] K.-K. Ni, S. Ospelkaus, M. H. G. de Miranda, A. Peér, B. Neyenhuis, J. J. Zirbel, S. Kotochigova, P. S. Julienne, D. S. Jin, and J. Ye, Science 322, 231 (2008).

[13] S. Ospelkaus, A. Pe'er, K. K. Ni, J. J. Zirbel, B. Neyenhuis, S. Kotochigova, P. S. Julienne, J. Ye, and D. S. Jin, Nat. Phys. 4, 622 (2008).

[14] S. Ospelkaus, K. K. Ni, M. H. G. de Miranda, A. Pe'er, B. Nyenhuis, D. Wang, S. Kotochigova, P. S. Julienne, D. S. Jin, and J. Ye, Faraday Discuss. 142, 351 (2009).
[15] S. Ospelkaus, K.-K. Ni, G. Quéméner, B. Neyenhuis, D. Wang, M. H. G. de Miranda, J. L. Bohn, J. Ye, and D. S. Jin, Phys. Rev. Lett. 104, 030402 (2010).

[16] A. Chotia, B. Neyenhuis, S. A. Moses, B. Yan, J. P. Covey, M. Foss-Feig, A. M. Rey, D. S. Jin, and J. Ye, Phys. Rev. Lett. 108, 080405 (2012).

[17] M. H. G. de Miranda, A. Chotia, B. Neyenhuis, D. Wang, G. Quemener, S. Ospelkaus, J. L. Bohn, J. Ye, and D. S. Jin, Nat. Phys. 7, 502 (2011).

[18] S. Ospelkaus, K.-K. Ni, D. Wang, M. H. G. de Miranda, B. Neyenhuis, G. Quéméner, P. S. Julienne, J. L. Bohn, D. S. Jin, and J. Ye, Science 327, 853 (2010).

[19] K. R. A. Hazzard, B. Gadway, M. Foss-Feig, B. Yan, S. A. Moses, J. P. Covey, N. Y. Yao, M. D. Lukin, J. Ye, D. S. Jin, and A. M. Rey, Phys. Rev. Lett. 113, 195302 (2014).

[20] B. Neyenhuis, B. Yan, S. A. Moses, J. P. Covey, A. Chotia, A. Petrov, S. Kotochigova, J. Ye, and D. S. Jin, Phys. Rev. Lett. 109, 230403 (2012).

[21] B. Yan, S. A. Moses, B. Gadway, J. P. Covey, K. R. A. Hazzard, A. M. Rey, D. S. Jin, and J. Ye, Nature (London) 501, 521 (2013).

[22] S. A. Moses, J. P. Covey, M. T. Miecnikowski, B. Yan, B. Gadway, J. Ye, and D. S. Jin, Science 350, 659 (2015).

[23] P. S. Żuchowski and J. M. Hutson, Phys. Rev. A 81, 060703 (2010).

[24] M.-S. Heo, T. T. Wang, C. A. Christensen, T. M. Rvachov, D. A. Cotta, J.-H. Choi, Y.-R. Lee, and W. Ketterle, Phys. Rev. A 86, 021602 (2012).

[25] S. Dutta, J. Lorenz, A. Altaf, D. S. Elliott, and Y. P. Chen, Phys. Rev. A 89, 020702 (2014).

[26] J. Deiglmayr, A. Grochola, M. Repp, K. Mörtlbauer, C. Glück, J. Lange, O. Dulieu, R. Wester, and M. Weidemüller, Phys. Rev. Lett. 101, 133004 (2008).

[27] J. Deiglmayr, A. Grochola, M. Repp, O. Dulieu, R. Wester, and M. Weidemüller, Phys. Rev. A 82, 032503 (2010).

[28] R. Barnett, D. Petrov, M. Lukin, and E. Demler, Phys. Rev. Lett. 96, 190401 (2006).

[29] A. V. Gorshkov, S. R. Manmana, G. Chen, J. Ye, E. Demler, M. D. Lukin, and A. M. Rey, Phys. Rev. Lett. 107, 115301 (2011).

[30] A. V. Gorshkov, S. R. Manmana, G. Chen, E. Demler, M. D. Lukin, and A. M. Rey, Phys. Rev. A 84, 033619 (2011).

[31] M. L. Wall and L. D. Carr, Phys. Rev. A 82, 013611 (2010).

[32] B. Zhu, B. Gadway, M. Foss-Feig, J. Schachenmayer, M. L. Wall, K. R. A. Hazzard, B. Yan, S. A. Moses, J. P. Covey, D. S. Jin, J. Ye, M. Holland, and A. M. Rey, Phys. Rev. Lett. 112, 070404 (2014).

[33] A. Micheli, Z. Idziaszek, G. Pupillo, M. A. Baranov, P. Zoller, and P. S. Julienne, Phys. Rev. Lett. 105, 073202 (2010).

[34] Z. Idziaszek, K. Jachymski, and P. S. Julienne, New J. Phys. 17, 035007 (2015).

[35] A. Simoni, S. Srinivasan, J.-M. Launay, K. Jachymski, Z. Idziaszek, and P. S. Julienne, New J. Phys. 17, 013020 (2015).

[36] P. K. Molony, P. D. Gregory, Z. Ji, B. Lu, M. P. Köppinger, C. R. Le Sueur, C. L. Blackley, J. M. Hutson, and S. L. Cornish, Phys. Rev. Lett. 113, 255301 (2014).

[37] T. Takekoshi, L. Reichsöllner, A. Schindewolf, J. M. Hutson, C. R. Le Sueur, O. Dulieu, F. Ferlaino, R. Grimm, and H.-C. Nägerl, Phys. Rev. Lett. 113, 205301 (2014). 
[38] J. W. Park, S. A. Will, and M. W. Zwierlein, New J. Phys. 17, 075016 (2015).

[39] J. W. Park, S. A. Will, and M. W. Zwierlein, Phys. Rev. Lett. 114, 205302 (2015).

[40] M. Guo, B. Zhu, B. Lu, X. Ye, F. Wang, R. Vexiau, N. Bouloufa-Maafa, G. Quéméner, O. Dulieu, and D. Wang, arXiv:1602.03947.

[41] E. Shuman, J. Barry, and D. DeMille, Nature (London) 467, 820 (2010).

[42] D. J. McCarron, E. B. Norrgard, M. H. Steinecker, and D. DeMille, New J. Phys. 17, 035014 (2015).

[43] M. T. Hummon, M. Yeo, B. K. Stuhl, A. L. Collopy, Y. Xia, and J. Ye, Phys. Rev. Lett. 110, 143001 (2013).

[44] A. L. Collopy, M. T. Hummon, M. Yeo, B. Yan, and J. Ye, New J. Phys. 17, 055008 (2015).

[45] M. Yeo, M. T. Hummon, A. L. Collopy, B. Yan, B. Hemmerling, E. Chae, J. M. Doyle, and J. Ye, Phys. Rev. Lett. 114, 223003 (2015).

[46] B. K. Stuhl, M. T. Hummon, M. Yeo, G. Quéméner, J. L. Bohn, and J. Ye, Nature (London) 492, 396 (2012).

[47] M. Zeppenfeld, B. G. Englert, R. Glöckner, A. Prehn, M. Mielenz, C. Sommer, L. D. van Buuren, M. Motsch, and G. Rempe, Nature (London) 491, 570 (2012).

[48] S. Chervenkov, X. Wu, J. Bayerl, A. Rohlfes, T. Gantner, M. Zeppenfeld, and G. Rempe, Phys. Rev. Lett. 112, 013001 (2014).

[49] C. Meng, A. P. P. van der Poel, C. Cheng, and H. L. Bethlem, Phys. Rev. A 92, 023404 (2015).

[50] M. Lemeshko, R. V. Krems, J. M. Doyle, and S. Kais, Mol. Phys. 111, 1648 (2013).

[51] F. Wang, X. He, X. Li, B. Zhu, J. Chen, and D. Wang, New J. Phys. 17, 035003 (2015).

[52] M. Gröbner, P. Weinmann, F. Meinert, K. Lauber, E. Kirilov, and H.-C. Nägerl, arXiv:1511.05044.

[53] M. Mayle, B. P. Ruzic, and J. L. Bohn, Phys. Rev. A 85, 062712 (2012).

[54] M. Mayle, G. Quéméner, B. P. Ruzic, and J. L. Bohn, Phys. Rev. A 87, 012709 (2013).

[55] J. F. E. Croft and J. L. Bohn, Phys. Rev. A 89, 012714 (2014).

[56] J. Herbig, T. Kraemer, M. Mark, T. Weber, C. Chin, H.-C. Nägerl, and R. Grimm, Science 301, 1510 (2003).

[57] J. G. Danzl, E. Haller, M. Gustavsson, M. J. Mark, R. Hart, N. Bouloufa, O. Dulieu, H. Ritsch, and H.-C. Nägerl, Science 321, 1062 (2008).

[58] J. G. Danzl, M. J. Mark, E. Haller, M. Gustavsson, R. Hart, J. Aldegunde, J. M. Hutson, and H.-C. Nägerl, Nat. Phys. 6, 265 (2010).

[59] G. Reinaudi, C. B. Osborn, M. McDonald, S. Kotochigova, and T. Zelevinsky, Phys. Rev. Lett. 109, 115303 (2012).

[60] S. Stellmer, B. Pasquiou, R. Grimm, and F. Schreck, Phys. Rev. Lett. 109, 115302 (2012).

[61] A. Frisch, M. Mark, K. Aikawa, S. Baier, R. Grimm, A. Petrov, S. Kotochigova, G. Quéméner, M. Lepers, O. Dulieu et al., Phys. Rev. Lett. 115, 203201 (2015).

[62] S. Jochim, M. Bartenstein, A. Altmeyer, G. Hendl, S. Riedl, C. Chin, J. H. Denschlag, and R. Grimm, Science 302, 2101 (2003).

[63] D. Jaksch, C. Bruder, J. I. Cirac, C. W. Gardiner, and P. Zoller, Phys. Rev. Lett. 81, 3108 (1998).
[64] A. Sanpera, M. Lewenstein, V. Ahufinger, B. Damski, A. Sen De, and U. Sen, Adv. Phys. 56, 243 (2007).

[65] A. M. Kaufman, B. J. Lester, and C. A. Regal, Phys. Rev. X 2, 041014 (2012).

[66] J. D. Thompson, T. G. Tiecke, A. S. Zibrov, V. Vuletić, and M. D. Lukin, Phys. Rev. Lett. 110, 133001 (2013).

[67] R. D. Levine, Molecular Reaction Dynamics (Cambridge University Press, Cambridge, England, 2005).

[68] T. A. Brody, J. Flores, J. B. French, P. A. Mello, A. Pandey, and S. S. M. Wong, Rev. Mod. Phys. 53, 385 (1981).

[69] G. E. Mitchell, A. Richter, and H. A. Weidenmüller, Rev. Mod. Phys. 82, 2845 (2010).

[70] C. H. Greene, A. R. P. Rau, and U. Fano, Phys. Rev. A 26, 2441 (1982).

[71] Z. Idziaszek and P. S. Julienne, Phys. Rev. Lett. 104, 113202 (2010).

[72] Z. Idziaszek, G. Quéméner, J. L. Bohn, and P. S. Julienne, Phys. Rev. A 82, 020703 (2010).

[73] S. Kotochigova, New J. Phys. 12, 073041 (2010).

[74] L.-M. Duan, Phys. Rev. Lett. 95, 243202 (2005).

[75] H. P. Büchler, Phys. Rev. Lett. 104, 090402 (2010).

[76] M. L. Wall and L. D. Carr, Phys. Rev. Lett. 109, 055302 (2012).

[77] M. L. Wall and L.D. Carr, Phys. Rev. A 87, 033601 (2013).

[78] F. Deuretzbacher, K. Plassmeier, D. Pfannkuche, F. Werner, C. Ospelkaus, S. Ospelkaus, K. Sengstock, and K. Bongs, Phys. Rev. A 77, 032726 (2008).

[79] We assume trap frequencies are the same for the bound states and the molecules because the polarizabilities and mass are approximately double those of the latter.

[80] See the Supplemental Material at http://link.aps.org/ supplemental/10.1103/PhysRevLett.116.135301 for a discussion of the numerical techniques used to obtain the twomolecule spectrum, the regularization of the two-molecule theory, the accounting of threshold scattering behavior within quantum defect theory and transition state theory, and references [81-83].

[81] C. J. Pethick and H. Smith, Bose-Einstein Condensation in Dilute Gases (Cambridge University Press, Cambridge, England, 2002).

[82] J. P. Burke Jr., Ph.D. thesis, University of Colorado, 1999.

[83] G. Gribakin and V. V. Flambaum, Phys. Rev. A 48, 546 (1993).

[84] K. Baumann, N. Q. Burdick, M. Lu, and B. L. Lev, Phys. Rev. A 89, 020701 (2014).

[85] T. Maier, I. Ferrier-Barbut, H. Kadau, M. Schmitt, M. Wenzel, C. Wink, T. Pfau, K. Jachymski, and P. S. Julienne, Phys. Rev. A 92, 060702 (2015).

[86] T. Maier, H. Kadau, M. Schmitt, M. Wenzel, I. FerrierBarbut, T. Pfau, A. Frisch, S. Baier, K. Aikawa, L. Chomaz, M. J. Mark, F. Ferlaino, C. Makrides, E. Tiesinga, A. Petrov, and S. Kotochigova, Phys. Rev. X 5, 041029 (2015).

[87] K. Jachymski and P. S. Julienne, Phys. Rev. A 92, 020702 (2015).

[88] K. Jachymski and P. S. Julienne, Phys. Rev. A 88, 052701 (2013).

[89] M. D. Frye, M. Morita, C. L. Vaillant, D. G. Green, and J. M. Hutson, arXiv:1511.08744. 
[90] B. P. Ruzic, C. H. Greene, and J. L. Bohn, Phys. Rev. A 87, 032706 (2013).

[91] P. S. Julienne, T. M. Hanna, and Z. Idziaszek, Phys. Chem. Chem. Phys. 13, 19114 (2011).

[92] J. von Stecher, V. Gurarie, L. Radzihovsky, and A. M. Rey, Phys. Rev. Lett. 106, 235301 (2011).

[93] C. Kollath, A. Iucci, I. P. McCulloch, and T. Giamarchi, Phys. Rev. A 74, 041604 (2006).

[94] R. Jördens, N. Strohmaier, K. Günter, H. Moritz, and T. Esslinger, Nature (London) 455, 204 (2008).
[95] S. D. Huber and A. Rüegg, Phys. Rev. Lett. 102, 065301 (2009).

[96] A. M. Kaufman, B. J. Lester, C. M. Reynolds, M. L. Wall, M. Foss-Feig, K. R. A. Hazzard, A. M. Rey, and C. A. Regal, Science 345, 306 (2014).

[97] B. J. Lester, N. Luick, A. M. Kaufman, C. M. Reynolds, and C. A. Regal, Phys. Rev. Lett. 115, 073003 (2015).

[98] T. M. Hanna, E. Tiesinga, W. F. Mitchell, and P. S. Julienne, Phys. Rev. A 85, 022703 (2012). 